\title{
IDENTIFICAÇÃO DOS BENEFÍCIOS E DIFICULDADES DA PRODUÇÃO MAIS LIMPA EM EMPRESAS INDUSTRIAIS DO ESTADO DE SÃO PAULO
}

\section{IDENTIFICATION OF CLEANER PRODUCTION BENEFITS AND DIFFICULTIES IN INDUSTRIAL ENTERPRISES OF SÃO PAULO}

\author{
José Augusto de Oliveira*E-mail:joseaugusto@sc.usp.br \\ Mariana Guardia E-mail: marianaguardia@gmail.com \\ Geandra Alves Queiroz* E-mail: geandraqueiroz@ymail.com \\ Raphael Laraia Rocha de Barros Cobra* E-mail: \\ raphaelcobra@gmail.com \\ Aldo Roberto Ometto* E-mail:aometto@sc.usp.br \\ Otávio José de Oliveira** E-mail: otaviodeoliveira@uol.com.br \\ *Universidade de São Paulo (USP), São Carlos, São Paulo \\ * Universidade Paulista Júlio de Mesquita Filho (UNESP), Guaratinguetá, São Paulo
}

\begin{abstract}
Resumo: A Produção mais Limpa está conquistando crescente aceitação pelas empresas devido ao potencial que esta estratégia apresenta para minimização proativa de impactos ambientais para a racionalização na utilização de insumos e diminuir a geração de resíduos e emissões. Esta prática gera benefícios ambientais e econômicos para as empresas. Desta forma, a mensuração dos benefícios auferidos pela Produção mais Limpa em empresas que adotam esta estratégia ambiental é de suma importância para a sua promoção no meio empresarial e também para a sociedade em geral. Somado a estas informações, nota-se a necessidade de maior valorização dos benefícios ambientais face aos econômicos. Neste contexto, esta pesquisa tem como objetivo identificar os benefícios gerados e as dificuldades enfrentadas para a Produção mais Limpa em quatro empresas industriais situadas no estado de São Paulo por meio de estudos de caso. Foi possível identificar significativos benefícios ambientais, principalmente no aspecto de redução da utilização de insumos de produção, ou seja, extração de recursos naturais e na deposição dos resíduos da produção. Além disso, a estratégia preventiva da Produção mais Limpa possibilitou ganhos econômicos às organizações, sustentados pelo gerenciamento racional de seus processos produtivos. Como principal dificuldade destaca-se o aspecto econômico para a implantação de projetos de Produção mais Limpa.
\end{abstract}

Palavras-chave: Produção mais Limpa. Benefícios. Dificuldades. Empresas Industriais. Estado de São Paulo.

Abstract: The Cleaner Production is gaining increasing acceptance by companies due the capacity that this strategy presents to proactive minimization of environmental impacts and to the rational use of inputs and reduce the generation of waste and emissions. This practice creates environmental and economic benefits for businesses. Thus, the measurement of the benefits earned by the Cleaner Production in companies that adopt this environmental strategy is of paramount importance to its promotion in business and for society in general. In addition to this information, there is a need for greater appreciation of the environmental benefits against the economic. In this context, this research aims to identify the benefits and the difficulties generated for Cleaner Production in four industrial companies located in the state of São Paulo through case studies. It was possible to identify significant environmental benefits, especially in the aspect of reducing the use of production inputs, i.e., extraction of natural resources and disposal of waste production. Moreover, the preventive strategy of Cleaner Production allwoed economic gains to organizations, supported by rational 
management of their production processes. As a major difficulty highlight the economic aspects for the implementation of projects Cleaner Production.

Keywords: Cleaner Production. Benefits. Difficulties. Industrial Companies. São Paulo State.

\section{INTRODUÇÃO}

As empresas buscam ações e medidas que as auxiliem na minimização dos impactos ambientas e na maximização dos lucros por meio de gerenciamento correto de seus meios de produção (SILVA; MEDEIROS, 2004; CHAVAN, 2005; CAGNO; TRUCCO; TARDINI, 2005; KLEMĚS; VARBANOV; HUISING, 2012; KUBOTA; CAMPOS; CAUCHICK MIGUEL, 2014).

Nota-se uma mudança significativa no cenário empresarial focada na adoção de procedimentos e medidas ambientais (FRYXELL; SZETO, 2002; YUSUP et al., 2014; KUBOTA, CAMPOS E CAUCHICK MIGUEL, 2014). Estas práticas refletem a maximização da eficiência produtiva e com isso ganhos econômicos para as empresas, aumentando suas condições de competitividade frente ao mercado (KILBOURNE, 2004).

Seguindo esta tendência, A Produção mais Limpa $(P+L)$ é "aplicação contínua de uma estratégia ambiental preventiva integrada a processos, produtos e serviços para aumentar a eficiência global e reduzir riscos para os seres humanos e o meio ambiente". A $\mathrm{P}+\mathrm{L}$ pode ser aplicada aos processos utilizados em toda a organização, aos próprios produtos, e de diversos serviços fornecidos na sociedade (UNEP, 2002; MACIEL; FREITAS, 2013).

Esta estratégia ambiental atua diretamente no gerenciamento da produção e proporciona significativos ganhos ambientais e econômicos às organizações, pois procura atuar na fonte geradora dos resíduos, sendo então uma estratégia ambientalmente preventiva e proativa de produção (FRYXELL; SZETO, 2002; UNEP, 2002; UNIDO; UNEP, 2011; KLEMĚS; VARBANOV; HUISING, 2012; SILVA et al., 2013; YUSUP et al., 2014).

A Organização das Nações Unidas (ONU), por meio de seu programa United Nations for Environment Programme (UNEP) deseja difundir a $\mathrm{P}+\mathrm{L}$ em várias escalas: no mundo, na América Latina e no Brasil. Os esforços de divulgação da $\mathrm{P}+\mathrm{L}$ foram divididos em iniciativas regionais dentre as quais o Programa de consumo 
e produção Sustentável da América Latina e Caribe é a vertente que conta com participação brasileira (PNUMA, 2014).

No Brasil, os principais apoiadores da $\mathrm{P}+\mathrm{L}$ é a Companhia Ambiental do Estado de São Paulo (CETESB) que tem o papel de divulgação regional do estado de São Paulo das práticas de $\mathrm{P}+\mathrm{L}$, além da documentação e divulgação de 90 casos de sucesso e o Centro Nacional de Tecnologias Limpas (CNTL) (CNTL, 2001; CETESB, 2014).

O recorte geográfico da pesquisa se limita ao estado de São Paulo que é responsável por 33\% do Produto Interno Bruto (PIB) brasileiro, sendo que 29\% deste PIB são resultantes da atividade industrial do estado (IBGE, 2009). Neste estado estima-se que são produzidos a cada ano cerca de 25 milhões de toneladas de resíduos industriais, sendo cerca 535 mil toneladas de resíduos perigosos. Desses resíduos perigosos apenas 53\% são tratados, 31\% armazenados e o restante 16\% são dispostos corretamente (CETESB; PNUMA, 2005).

A $\mathrm{P}+\mathrm{L}$ está conquistando crescente aceitação pelas empresas por caracterizar-se pela minimização proativa de impactos ambientais e por racionalizar a utilização de insumos e diminuir a geração de resíduos e emissões. Esta prática gera benefícios ambientais e econômicos para as empresas. Desta forma, a mensuração dos benefícios auferidos pela $\mathrm{P}+\mathrm{L}$ em empresas que adotam esta estratégia ambiental é de suma importância para a sua promoção no meio empresarial e também para a sociedade em geral (HILSON, 2000; MITCHEL, 2005; VAN HOOF; LYON, 2013; MACIEL; FREITAS, 2013).

A proposta do presente trabalho é avaliar projetos e procedimentos de $\mathrm{P}+\mathrm{L}$, que diferem dos casos apresentados na literatura devido a existências de longos prazos que faz com que estes sejam avaliados de forma mais ampla e conjunta. A importância desse tipo de avaliação está na verificação de engajamento de escala dos projetos de $\mathrm{P}+\mathrm{L}$.

Deste modo, o objetivo principal desta pesquisa foi identificar, por meio de estudos de caso, os benefícios ambientais e econômicos oriundos da implantação de projetos e procedimentos de $\mathrm{P}+\mathrm{L}$ e as dificuldades para a implementação desta estratégia em quatro empresas industriais brasileiras situadas no estado de São Paulo. 
Na seção seguinte é feita a apresentação da revisão bibliográfica sobre o tema $\mathrm{P}+\mathrm{L}$. Após esta etapa é demonstrado o método de pesquisa utilizado e os estudos de casos. O trabalho é finalizado pelas conclusões finais e pela apresentação das referências utilizadas.

\section{REFERENCIAL TEÓRICO}

Após casos de sucesso na redução de impactos ambientais e custos inspirados principalmente pela empresa norte americana 3M com o seu programa 3P (Pollution Prevention Pays), o UNEP estruturou e passou a difundir a $\mathrm{P}+\mathrm{L}$. Esta foi definida pelo UNEP como a aplicação contínua de uma estratégia ambiental preventiva integrada a processos, produtos e serviços para aumentar a eficiência global e reduzir riscos para os seres humanos e o meio ambiente. A $P+L$ pode ser aplicada aos processos utilizados em toda a empresa, aos próprios produtos, e de diversos serviços fornecidos na sociedade (UNEP, 1995; UNIDO/ UNEP, 2011; UNEP, 2002; ELIAS; MAGALHÃES, 2003).

$A P+L$ objetiva principalmente a prevenção à poluição, distinguindo assim de outros programas, sistemas e ferramentas ambientais focados em medidas de fimde-tubo, utilizados antes da sua criação (UNEP, 2002; BASS, 2007; RIAZ; ZAHEDI; KLEMĚS, 2013; VAN HOOF; LYON, 2013). Para isso, a $\mathrm{P}+\mathrm{L}$ atua especialmente em duas macro categorias:

- minimização de materiais realizada pela reciclagem interna ou pela redução na fonte; e

- reutilização de materiais, que é feita pela reciclagem externa ao processo produtivo (CNTL, 2001).

O programa mais parecido com a $\mathrm{P}+\mathrm{L}$, muito utilizado por países da América do Norte chama-se Pollution Prevention, mundialmente conhecido como P2. Segundo UNEP (2002), a P+L e a P2 eles possuem basicamente a mesma estrutura e os mesmos objetivos, e muitas vezes são utilizadas de forma intercambiável na literatura (UNIDO/ UNEP, 2004). Contudo, Hilson (2003) e UNIDO/UNEP (2004) afirmam que a $\mathrm{P}+\mathrm{L}$ é mais abrangente em termos de aplicação e de escopo do que a P2. 
O programa de $\mathrm{P}+\mathrm{L}$ lançado pelo UNEP é disseminado em vários países do mundo por meio de centros de apoio e difusão da estratégia. No Brasil, CNTL situado no Rio Grande do Sul, vem desde 1996 promovendo a $\mathrm{P}+\mathrm{L}$ pelo setor empresarial. A CETESB oferece suporte técnico a administrativo para a $\mathrm{P}+\mathrm{L}$ no estado de São Paulo (CETESB; PNUMA, 2005).

Alguns países em desenvolvimento como a China trazem estudos de caso expressivos (ZHI-DONG , SHU-SHEN , et al., 2011; HUANG , JIWEN e BIN , 2013). O caso apresentado por Huang Jwen e Bin (2013) estuda a indústria cerâmica da província de Guangdong na China. A $\mathrm{P}+\mathrm{L}$ nesse caso foi combinada a auditorias periódicas e proporcionou, para cada unidade produzida, a redução de 4,3\% no consumo de energia, $22,33 \%$ do consumo de água, $8 \%$ na emissão de $\mathrm{CO}_{2}$. As principais práticas de $\mathrm{P}+\mathrm{L}$ empregadas foram a substituição das instalações, troca de tecnologia, controle de processos, reutilização de matérias primas e resíduos, melhorias de gestão e treinamento de funcionários.

O caso de $\mathrm{P}+\mathrm{L}$ em organizações farmacêuticas chinesas descrito por Shushen et al. (2011) apresenta a redução de do uso de carvão de 2,2 \% e 1,53\% de água aliados a diminuição de resíduos em 3,1\% e grande redução no uso de insumos. Para esse caso foram empregados o desenvolvimento de processos, a atualização de equipamentos, a exploração de fluxogramas, o uso de fonte de energia mais limpa, a inclusão de reciclagem nos processos de manufatura, a reciclagem de desperdícios, melhorias de gestão e a garantia de disposição correta de resíduos.

O caso Chileno de Rivela et al. (2004), aborda um curtume chileno que a partir de uma análise de entradas e saídas (balanço de massa) foi dada prioridade para otimização do tratamento de efluentes. Um estudo brasileiro de Kirst, Moutaqui e Machado (2009) trata de um abatedouro de aves no qual foi estudado o Layout, fluxogramas quantitativos e qualitativos, feita a avaliação dos impactos em estágios críticos do processo e avaliação de indicadores. Foi dada mais atenção aos processos de geração de efluentes e as medidas adotadas evitaram o lançamento de efluentes com alto teor de gordura e baixo oxigênio dissolvido.

Além desse tipo de foco o trabalho de Lima (2012), estuda opções para reuso dos resíduos gerados por artesãos de pedra sabão na região de Ouro Preto, encontrando usos em filtros de inseticidas e papeis de parede e embalagens. 
Para Fresner (1998), Hicks e Dietmar (2007), Oliveira (2011) e Yusup et al. (2014), a P+L se apresenta como uma estratégia importante para minimizar e prevenir impactos ambientais adversos da produção industrial em escalas cada vez mais crescentes.

A adoção da P+L está sendo muito bem vista por acionistas e stakeholders. Em muitas empresas, esta estratégia ambiental é adotada segundo a sua contribuição importante para a minimização dos impactos ambientais decorrentes da manufatura e também como medida para reduzir custos e maximizar lucros (YÜKSEL, 2008; OLIVEIRA, 2011; Yusup et al., 2014).

As duas macro categorias iniciais de abrangência da $P+L$ se desdobram em níveis de aplicação, onde são determinados seus focos pontuais.

A Figura 1 apresenta esta divisão e estratificação de focos de atuação da $\mathrm{P}+\mathrm{L}$.

Figura 1 - Níveis de aplicação da Produção Mais Limpa

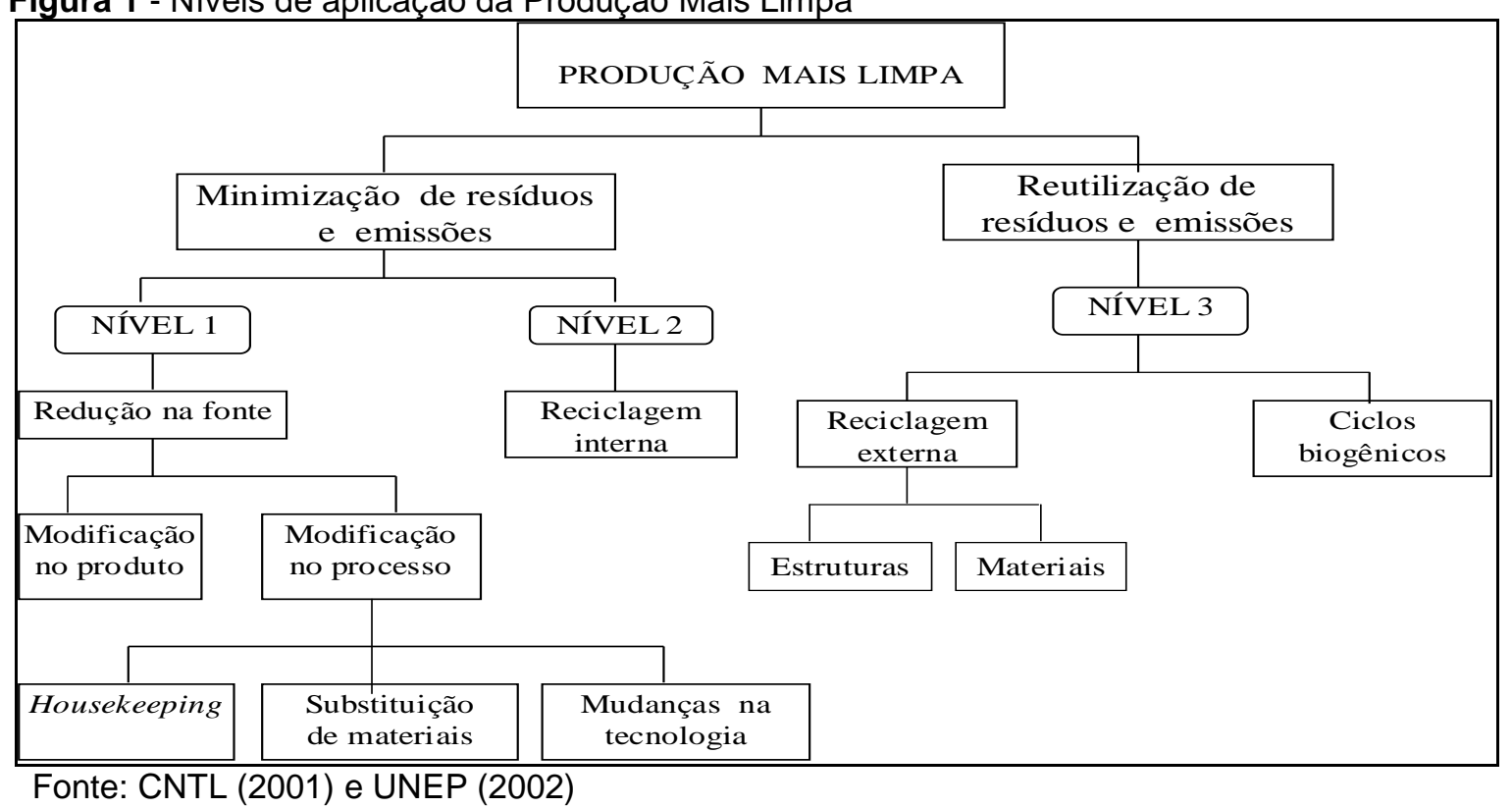

Segundo UNEP (2002), a P+L é implantada sem cinco fases contendo passos específicos a serem seguidos neste processo, são eles:

- Planejamento e organização - Nesta fase realizam-se os passos comprometimento da gerência; definição do Ecotime; formulação dos objetivos e metas; e identificação das barreiras; 
- Pré-avaliação - Nesta fase realizam-se os passos elaboração do fluxograma do processo; avaliação das entradas e saídas e determinação dos focos da avaliação de $\mathrm{P}+\mathrm{L}$;

- Avaliação - Nesta fase realizam-se os passos balanço de material; avaliação das causas; identificação das oportunidades de P+L; e seleção das oportunidades de $\mathrm{P}+\mathrm{L}$;

- Estudo da viabilidade - Nesta fase realizam-se os passos avaliação preliminar; avaliação técnica; avaliação econômica; avaliação ambiental; e seleção das oportunidades;

- Implementação e monitoramento - Nesta fase realizam-se os passos plano de $\mathrm{P}+\mathrm{L}$; implementação de oportunidades de $\mathrm{P}+\mathrm{L}$; monitoramento e avaliação; e sustentação das atividades de $\mathrm{P}+\mathrm{L}$.

Para Lemos (1998), UNEP (2002), Barbieri (2007), Oliveira (2011) e a $P+L$ pode gerar importantes benefícios para as empresas que a adotam, os quais merecem destaque:

- Melhoria da imagem da empresa;

- Minimização e mitigação dos impactos ambientais gerados pela produção;

- Melhoria organizacional e da qualidade do trabalho;

- Aumento da ecoeficiência produtiva;

- Atendimento legal;

- Vantagem competitiva; e

- Redução de custos, economia financeira e aumento dos lucros.

A identificação dos principais benefícios da $P+L$ para as empresas pode contribuir para a disseminação desta estratégia ambiental no meio acadêmico e empresarial. As dificuldades e os benefícios apresentados demonstram que a P+L é viável ambientalmente, tecnicamente e economicamente, aumentando assim a sua aceitabilidade pelas organizações e o interesse por pesquisas científicas sobre o tema (HILSON, 2000; MITCHEL, 2005; YOUNGBLOOD et al., 2008).

Segundo Barbieri (2007) e Oliveira (2011), a P+L é uma estratégia diferente do Sistema de Gestão Ambiental (SGA), embora ambos possuam similaridades organizacionais e operacionais. Com isso, muitos benefícios, em empresas que possuem tanto a $\mathrm{P}+\mathrm{L}$ como o SGA de forma operante, são muitas vezes mensurados e atribuídos para ambos. 
A despeito dos significativos benefícios que a $\mathrm{P}+\mathrm{L}$ pode gerar para as organizações, a sua implantação e prática ainda enfrentam barreiras e dificuldades nas empresas, que segundo EPA (2001), SENAI (2003) e UNEP (2004), merecem destaque:

-Resistência à mudança;

-Falta de informação sobre a P+L;

-Falta de priorização para esta estratégia no negócio da empresa;

-Pressão para resultados em curto prazo;

-Desconfiança sobre o retorno do investimento;

-Não contabilização dos custos e dos benefícios ambientais;

-Baixa disponibilização de tempo e de conhecimentos técnicos;

-Baixa disponibilização de recursos;

-Falsa expectativa para a P+L sobre produtividade e a qualidade do produto;

-Falta de estrutura e políticas ambientais na empresa.

Segundo EPA (2001), identificar e reconhecer estas barreiras é um importante caminho em busca da superação destas dificuldades. Neste sentido, o envolvimento e efetivo comprometimento dos gestores é um elemento fundamental para o sucesso das práticas de $\mathrm{P}+\mathrm{L}$ nas empresas.

\section{MÉTODOS DE PESQUISA}

A pesquisa é um processo sistemático que busca encontrar respostas aos questionamentos que são propostos (GIL, 1991). Segundo Fleury (2012) a escolha do método de pesquisa é fundamental na condução deste processo para que o resultado final seja qualificado como pesquisa científica. O procedimento deste estudo pode ser visualizado na Figura 2.

Esta pesquisa tem o caráter exploratório, e segundo Gil (1991), este tipo de estudo tem como objetivo principal expor concepções de problemas reais e então contribuir para a construção do campo do conhecimento. Ainda, este tipo de pesquisa realiza uma busca na literatura para aprofundamento no tema, buscando identificar exemplos que auxiliem na compreensão da questão de pesquisa.

Com relação à abordagem da pesquisa, este estudo foi baseado em análises qualitativas. Este tipo de abordagem permite um refinamento das informações 
obtidas na revisão bibliográfica (VOSS; TSIKRIKTSIS; FROHLICH, 2002; YIN, 2005) e também a identificação de problemas e características reais (ZANELLI, 2002).

O método de estudos de caso é um importante meio para o desenvolvimento de uma pesquisa qualitativa, pois ele possui caráter exploratório e aproxima o pesquisador das evidências empíricas (YIN, 2005; JUPP, 2006).

Para Yin (2005) e Cauchick-Miguel (2007), os estudos de caso possuem uma função de exploração de informações teóricas em suas atividades práticas, possibilitando assim a confirmação e confronto da revisão bibliográfica com a realidade.

Nesta pesquisa optou-se pela adoção de múltiplos casos. A escolha de mais de estudo de caso justifica-se pelo fato de que esta pesquisa tem como objetivo ultrapassar a unicidade, pois segundo Yin (2005) as provas resultantes de casos múltiplos são consideradas mais convincentes e o estudo pode ser considerado mais robusto e adequado à realidade do fenômeno estudado.

Segundo Chauchick Miguel (2007) uma das primeiras tarefas para planejar um estudo de caso é a escolha da unidade de análise. Esta pesquisa foi baseada em 4 (quatro) casos, empresas Alfa, Beta, Gama e Delta. A escolha dos casos foi realizada segundo a caracterização feita pela CETESB (2010) das empresas como casos de sucesso em $\mathrm{P}+\mathrm{L}$. Posteriormente, foram selecionadas organizações que se propuseram a disponibilizar detalhes específicos de projetos e procedimentos de $\mathrm{P}+\mathrm{L}$ por meio de contatos prévios com o objetivo de identificar a disponibilidade de fornecimento de informações por parte dos responsáveis pela $\mathrm{P}+\mathrm{L}$. Segundo Voss, Tsikristsis e Frohlich (2002), a disponibilização de dados é essencial para o sucesso dos estudos de caso.

A investigação em projetos e procedimentos selecionados de $P+L$ foi proposta com o intuito de identificar-se os benefícios e as dificuldades específicas para cada um e não para programas mais amplos e gerais de $\mathrm{P}+\mathrm{L}$ ou ambientais nas empresas. A Figura 2 apresenta o fluxo do método de pesquisa realizado no trabalho. 
Figura 2 - Fluxo metodológico da pesquisa

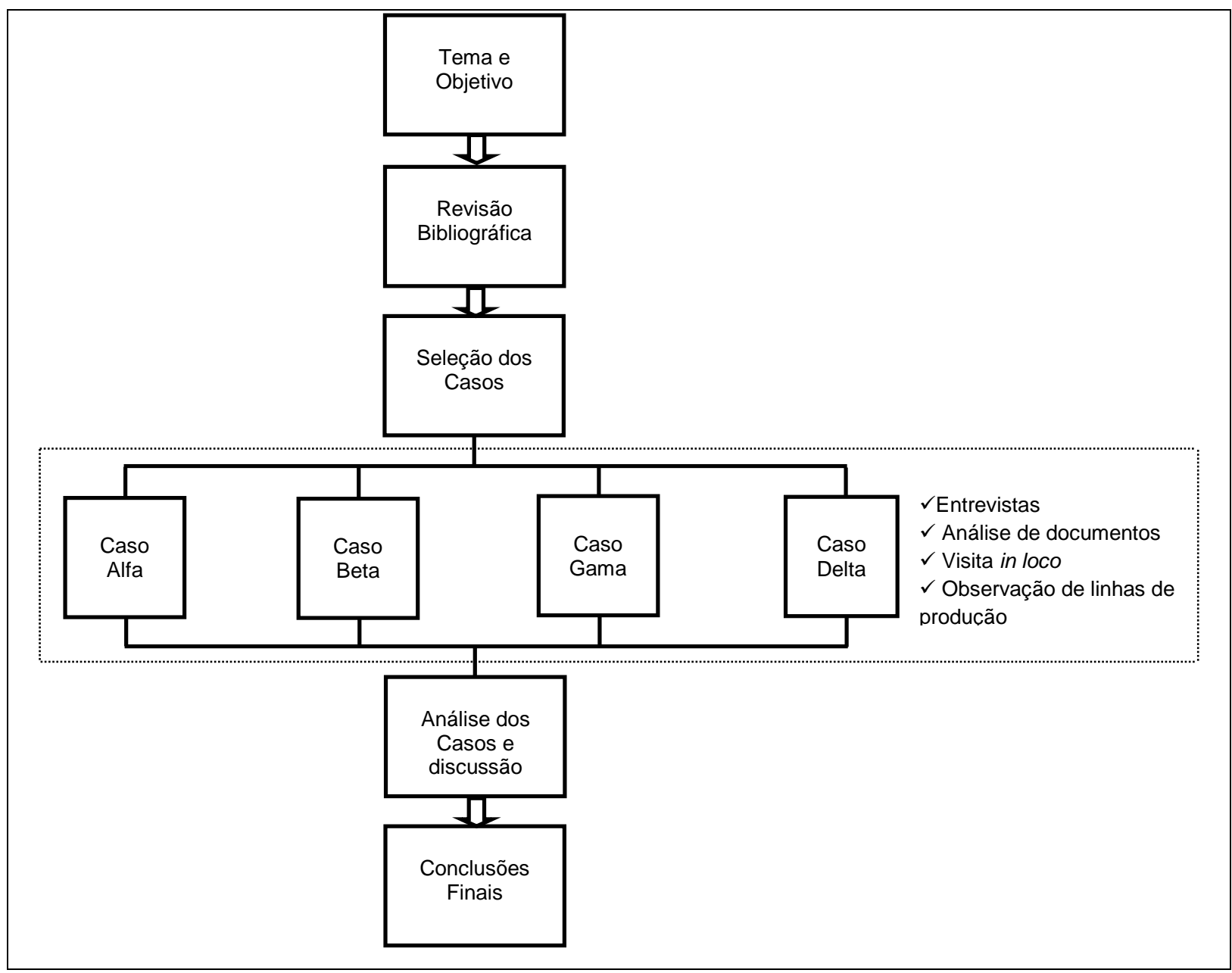

A utilização de mais de um instrumento de coleta de dados visa minimizar os vieses particulares de cada instrumento e com isso aumentar a veracidade das informações coletadas (CRESWELL, 1994; YIN, 2005).

As visitas in loco foram direcionadas por roteiros de entrevistas elaborados com base nos principais pontos da $\mathrm{P}+\mathrm{L}$ observados na revisão bibliográfica. Além das entrevistas, foram analisadas as principais linhas de produção das empresas para observação dos seus processos produtivos dos projetos e procedimentos de $\mathrm{P}+\mathrm{L}$, seguindo o protocolo de pesquisa apresentado pelo Quadro 1. 
Quadro 2 - Protocolo de estudo de caso

\begin{tabular}{|c|c|}
\hline $\begin{array}{l}\text { Questão de } \\
\text { pesquisa }\end{array}$ & $\begin{array}{l}\text { Quais são os benefícios e as dificuldades para a implantação de projetos e } \\
\text { procedimentos de } P+L \text { em empresas do ramo industrial brasileiras? }\end{array}$ \\
\hline $\begin{array}{l}\text { Unidade de } \\
\text { análise }\end{array}$ & $\begin{array}{l}\text { Representantes da Direção/ Engenheiros Ambientais e Responsáveis pelo } \\
\text { departamento de Meio Ambiente }\end{array}$ \\
\hline Local & $\begin{array}{l}\text { Quatro empresas industriais brasileiras casos de sucesso em P+L (Alfa, Beta, } \\
\text { Gema Delta) }\end{array}$ \\
\hline $\begin{array}{l}\text { Validade dos } \\
\text { construtos }\end{array}$ & $\begin{array}{l}\text { Identificação de adoção de projetos e procedimentos de } \mathrm{P}+\mathrm{L} \text { e avaliação dos } \\
\text { seus benefícios e de suas dificuldades }\end{array}$ \\
\hline Validade interna & $\begin{array}{l}\text { Análises realizadas por uma diversa gama ações como: entrevistas, análises de } \\
\text { documentos (certificados, projetos escritos, manuais de operações, } \\
\text { cronogramas de P+L e mapas de produção), arquivos internos das empresas e } \\
\text { visitas aos processos produtivos }\end{array}$ \\
\hline $\begin{array}{l}\text { Questões de } \\
\text { entrevistas dos } \\
\text { estudos de } \\
\text { casos }\end{array}$ & $\begin{array}{l}\text { - Caracterização geral da empresa; } \\
\text { - Caracterização do SGA da empresa; } \\
\text { - Caracterização e identificação de adoção de procedimentos de } P+L \text { nas } \\
\text { empresas; } \\
\text { - Etapas da implementação de P+L realizadas pelas empresas; e } \\
\text { - Convergências/ fomentos entre SGA (ISO 14001) e P+L e vice-versa. }\end{array}$ \\
\hline
\end{tabular}

As entrevistas foram realizadas com Representantes da Direção (RD) do SGA ISO 14001 e/ou responsáveis pelo departamento de Meio Ambiente das empresas. Estes funcionários foram considerados os mais adequados a responderem a entrevista por conduzirem os programas de $\mathrm{P}+\mathrm{L}$ nas empresas. As análises foram realizadas com base no método de Graneheim e Lundman (2004) e seguindo os passos recomendados por Yin (2005).

A análise documental foi realizada em projetos de $P+L$ e relatórios internos das empresas referentes aos projetos e procedimentos de $\mathrm{P}+\mathrm{L}$ implantados. Além disso, o manual do SGA ISO 14001 foi analisado por conter menções e detalhes sobre estes projetos e procedimentos.

\section{RESULTADOS E DISCUSSÃO}

Nesta seção são apresentados os casos estudados, iniciando-se pela caracterização das empresas, os benefícios da $\mathrm{P}+\mathrm{L}$, qualitativos e quantitativos e por fim, as principais dificuldades enfrentadas para a adoção da $\mathrm{P}+\mathrm{L}$.

Todas as empresas estudadas são do ramo industrial e de grande porte. Elas estão situadas no estado de São Paulo e são indicadas pela CETESB (2010) como casos de sucesso em $\mathrm{P}+\mathrm{L}$. 
Por motivos de confidencialidade seguindo o contrato estabelecido entre os pesquisadores e as empresas pesquisadas, suas razões sociais e demais informações que possibilitariam suas identificações foram mantidas em sigilo, o que não compromete a apresentação dos resultados obtidos.

Sendo assim, atribuíram-se nomes fictícios (Alfa, Beta, Gama e Delta) às organizações para apresentação de seus resultados no presente trabalho. A Tabela 1 apresenta a caracterização da amostra de estudo.

Tabela 1 - Caracterização geral das empresas da amostra da pesquisa

\begin{tabular}{|c|c|c|c|c|}
\hline Empresas & Alfa & Beta & Gama & Delta \\
\hline Setor de atuação & Químico & $\begin{array}{l}\text { Papel e } \\
\text { Celulose }\end{array}$ & Diversos & $\begin{array}{l}\text { Papel e } \\
\text { Celulose }\end{array}$ \\
\hline Faturamento (milhões de Euros em 2009) & 1,9 & 3067 & 648,43 & ---- \\
\hline $\mathrm{N}^{\circ}$ colaboradores & 3486 & 14600 & 3444 & 450 \\
\hline Consultoria na implantação do SGA ISO 14001 & Não & Não & Não & Sim \\
\hline Possuía um SGA antes da ISO 14001? & Sim & Sim & Sim & Sim \\
\hline Ano da última certificação ISO 9001 & 2009 & 2000 & 2010 & ---- \\
\hline Ano da última certificação ISO 14001 & 2009 & 2004 & 2009 & 2010 \\
\hline Operacionalização integrada do SGA e da $\mathrm{P}+\mathrm{L}$ & Não & Não & Sim & Sim \\
\hline
\end{tabular}

Observa-se pelo exposto que todas as organizações possuem a certificação ISO 14001, o que contribuiu significativamente para a implantação dos procedimentos de $\mathrm{P}+\mathrm{L}$. Contudo, as 4 (quatro) empresas operacionalizam o SGA e a $\mathrm{P}+\mathrm{L}$ de maneiras distintas. Assim, torna-se possível identificar e mensurar seus indicadores separadamente.

A empresa Alfa possui um departamento específico de Meio Ambiente, contudo, os projetos de $\mathrm{P}+\mathrm{L}$ são gerenciados pelos responsáveis de produção de cada processo. Já na organização Beta, os procedimentos de $P+L$ são liderados e gerenciados pelo departamento de Meio Ambiente que é o mesmo que supervisiona o SGA. Na empresa Gama, os projetos de P+L são, na maioria das vezes, criado pelos próprios funcionários que executam os processos de manufatura e do chão de fábrica, contando com o apoio de uma equipe (Staff) que pode auxiliar na sugestão e implementação de projetos de $\mathrm{P}+\mathrm{L}$. Na empresa $\mathrm{D}$, o departamento ambiental é responsável pela implementação da $\mathrm{P}+\mathrm{L}$ juntamente com o SGA.

Sob uma análise qualitativa confrontada com a literatura existente em $\mathrm{P}+\mathrm{L}$, foi possível observar alguns benefícios auferidos pela $\mathrm{P}+\mathrm{L}$ às empresas pesquisadas que são apresentados no Quadro 2. 
Quadro 2- Benefícios qualitativos identificados

\begin{tabular}{|l|c|c|c|c|}
\hline \multicolumn{1}{|c|}{ Benefícios } & \multicolumn{5}{c|}{ Empresas } \\
\hline Melhoria da imagem da empresa & Alfa & Beta & Gama & Delta \\
\hline Minimização dos impactos ambientais & $\checkmark$ & & & \\
\hline Redução dos custos de produção & $\checkmark$ & $\checkmark$ & $\checkmark$ & $\checkmark$ \\
\hline Agregação de valores aos resíduos & $\checkmark$ & $\checkmark$ & & \\
\hline Melhoria na cultura organizacional sobre o meio ambiente & $\checkmark$ & $\checkmark$ & $\checkmark$ & $\checkmark$ \\
\hline Minimização de custos e passivos ambientais & $\checkmark$ & $\checkmark$ & & $\checkmark$ \\
\hline Melhoria da comunicação com a sociedade & $\checkmark$ & & & \\
\hline Melhoria no controle e gerenciamento da poluição & $\checkmark$ & & & $\checkmark$ \\
\hline Auxílio em demais certificações da empresa & & $\checkmark$ & $\checkmark$ & $\checkmark$ \\
\hline Redução na utilização de matéria-prima & & $\checkmark$ & & \\
\hline Melhoria do controle de documentos e processos & & & $\checkmark$ & $\checkmark$ \\
\hline
\end{tabular}

Os principais benefícios identificados e em destaque no Quadro 2 foram:

- minimização dos impactos ambientais decorrentes das atividades produtivas, pois a $\mathrm{P}+\mathrm{L}$ previne a poluição e controla outros impactos ambientais durante a manufatura;

- redução dos custos de produção devido à racionalização e melhoria no gerenciamento de insumos de produção;

- agregação de valores aos resíduos, pois estes são reutilizados e reciclados dentro e fora dos sistemas de produção, possibilitando diminuição na utilização de novos subprodutos e economia com o tratamento de resíduos;

- melhoria na cultura organizacional sobre o meio ambiente já que a prática da P+L dissemina a conscientização ambiental dos funcionários, especialmente se forem estimulados por programas motivacionais para a inovação e adoção de melhorias no sistema produtivo como ocorre nas duas organizações estudadas;

- melhoria no gerenciamento e controle da poluição; e

- a redução no consumo de matéria prima.

A padronização no controle de documentos não é estritamente necessária para a implantação de projetos de $\mathrm{P}+\mathrm{L}$, esta então pode ser uma razão para a não constatação da melhoria no controle de documentos nas empresas e também prevista pela literatura.

Estes indicadores qualitativos são importantes instrumentos para a estimulação das empresas à adoção de práticas de $\mathrm{P}+\mathrm{L}$. Alguns benefícios importantes da $\mathrm{P}+\mathrm{L}$ às organizações que são previstos pela literatura não foram 
observados robustamente em todos os estudos de caso, mas foram identificados de forma isolada em alguns deles. A falta de maior aprofundamento nestes benefícios mostrados no Quadro 2 não significa falta de importância à estes, mas procurou-se nesta análise discutir os benefícios que foram encontrados em um maior número dos casos, seguindo os princípios da síntese cruzada dos casos (YIN, 2005).

Além destes benefícios, as duas empresas mensuram os indicadores ambientais e econômicos dos procedimentos de $\mathrm{P}+\mathrm{L}$. Estes benefícios são ilustrados no Quadro 3.

Quadro 3 - Benefícios quantitativos mensurados

\begin{tabular}{|c|c|c|c|}
\hline & Projeto & Benefícios Ambientais & Benefícios Econômicos \\
\hline \multirow{3}{*}{$\frac{\pi}{4}$} & $\begin{array}{l}\text { Tratamento e reutilização de } \\
\text { substâncias químicas utilizadas } \\
\text { em processos produtivos }\end{array}$ & $\begin{array}{l}\text { Redução da necessidade, do } \\
\text { descarte e da deposição dos resíduos } \\
\text { do insumo químico (quantidade não } \\
\text { calculada) }\end{array}$ & $\begin{array}{l}\text { Economia de US\$0,76 } \\
\text { milhões/ano em cada } \\
\text { processo produtivo }\end{array}$ \\
\hline & $\begin{array}{l}\text { Programas de treinamento, } \\
\text { conscientização e motivação } \\
\text { para melhorias contínuas no } \\
\text { gerenciamento dos processos } \\
\text { produtivos }\end{array}$ & $\begin{array}{l}\text { Redução de cerca de } 300 \text { toneladas } \\
\text { de resíduos de } 2002 \text { a } 2003 \text { nos } \\
\text { processos produtivos da empresa }\end{array}$ & $\begin{array}{l}\text { Economia de US } \$ 379,84 \\
\text { mil de } 2002 \text { a } 2003 \text { nos } \\
\text { processos produtivos da } \\
\text { empresa }\end{array}$ \\
\hline & $\begin{array}{l}\text { Utilização de centrífuga para a } \\
\text { separação de resíduos sólidos } \\
\text { de resíduos líquidos, } \\
\text { possibilitando assim a } \\
\text { reutilização destes resíduos }\end{array}$ & $\begin{array}{l}\text { Redução da demanda de material e } \\
\text { Eliminação do descarte dos resíduos } \\
\text { produtivos da empresa (quantidade } \\
\text { não calculada) }\end{array}$ & $\begin{array}{l}\text { Economia de US\$ } 316,54 \\
\text { mil/ ano nos processos } \\
\text { produtivos da empresa }\end{array}$ \\
\hline \multirow{3}{*}{ 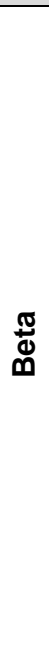 } & $\begin{array}{l}\text { Queima de biomassa resultante } \\
\text { de resíduos de madeira e } \\
\text { celulose. Esta energia térmica é } \\
\text { convertida em energia elétrica e } \\
\text { supre as necessidades da } \\
\text { planta da empresa }\end{array}$ & $\begin{array}{l}\text { Redução do uso de combustível fóssil } \\
\text { e da geração de } 30 \% \text { de resíduos do } \\
\text { processo }\end{array}$ & $\begin{array}{l}\text { Redução de custos US } \$ \\
417.083 .00 / \text { ano para a } \\
\text { compra de GN e óleo }\end{array}$ \\
\hline & $\begin{array}{l}\text { Diminuição da utilização de } \\
\text { insumos produtivos de celulose } \\
\text { em todo o processo por meio } \\
\text { de: } \\
\text { •Cozimento modificado; } \\
\text {-Deslignificação com ozônio; e } \\
\text { Branqueamento com ozônio. }\end{array}$ & $\begin{array}{l}\text { Redução da Geração Dregs+Gritz: } \\
\text { 35\%; } \\
\text { Redução da Geração Lama: 55\%; } \\
\text { Reutilização da lama gerada: 50\%; e } \\
\text { Reciclagem da lama gerada: } 50 \%\end{array}$ & $\begin{array}{l}\text { Economia de US\$ } \\
208.028 .00 / \text { ano no } \\
\text { processo de produção de } \\
\text { papel }\end{array}$ \\
\hline & $\begin{array}{l}\text { Tratamento em ciclos fechados } \\
\text { de produção e reutilização de } \\
\text { substâncias químicas utilizadas } \\
\text { no branqueamento da celulose } \\
\text { e papel }\end{array}$ & $\begin{array}{l}\text { Redução da demanda dos produtos e } \\
\text { em seus descartes, diminuindo } \\
\text { consideravelmente a necessidade de } \\
\text { tratamento em ETE e suas emissões } \\
\text { aos cursos d'água }\end{array}$ & $\begin{array}{l}\text { Economia de US\$ } \\
\text { 151.094.00/ ano no } \\
\text { processo da madeira }\end{array}$ \\
\hline 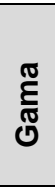 & $\begin{array}{l}\text { Impressão em placas e outros } \\
\text { por meio digital, eliminando } 4 \\
\text { das } 8 \text { etapas da manufatura, } \\
\text { sendo que } 2 \text { dessas } 4 \text { etapas, } \\
\text { emitiam gases nocivos }\end{array}$ & $\begin{array}{l}\text { •Redução da emissão de } \\
\text { gases estufantes; } \\
\text { •Econômica de energia; }\end{array}$ & $\begin{array}{l}\text { Economia na utilização } \\
\text { de gás natural (etapa do } \\
\text { processo suprida) e } \\
\text { otimização da produção }\end{array}$ \\
\hline
\end{tabular}


Quadro 3 - Benefícios quantitativos mensurados

(conclusão)

\begin{tabular}{|c|c|c|c|}
\hline & Projeto & Benefícios Ambientais & Benefícios Econômicos \\
\hline & $\begin{array}{l}\text { Troca de caixas de transporte } \\
\text { de madeira não retornáveis por } \\
\text { caixas metálicas retornáveis }\end{array}$ & $\begin{array}{l}\text { Redução de } 400 \\
\text { toneladas de madeira por } \\
\text { ano }\end{array}$ & Não informado \\
\hline & $\begin{array}{l}\text { Redução de atividades } \\
\text { logísticas desnecessárias e sem } \\
\text { valores agregados }\end{array}$ & $\begin{array}{l}\text { Redução da utilização de } \\
\text { combustíveis e emissão } \\
\text { de gases (não } \\
\text { quantificada) }\end{array}$ & $\begin{array}{l}\text { Economia de } \mathrm{R} \$ 60711,00 \\
\text { por ano }\end{array}$ \\
\hline & $\begin{array}{l}\text { Substituição de embalagens de } \\
\text { papelão para polímeros por } \\
\text { embalagens realizadas do } \\
\text { mesmo polímero, que é } \\
\text { incorporado ao produto }\end{array}$ & $\begin{array}{l}\text { Redução de deposição de } \\
24,5 \text { toneladas por ano de } \\
\text { papelão }\end{array}$ & $\begin{array}{l}\text { Redução de } 54 \% \text { do } \\
\text { custo das embalagens }\end{array}$ \\
\hline & $\begin{array}{l}\text { Criação de esponja de limpeza } \\
\text { realizada com matéria natural e } \\
\text { polímero reciclável }\end{array}$ & $\begin{array}{l}\text {-Redução de } 44 \% \text { na utilização de } \\
\text { recursos; } \\
\text {-Redução de } 52 \% \text { do uso de energia } \\
\text { para a produção de esponjas; } \\
\text {-Redução de } 23 \% \text { do uso de água na } \\
\text { produção de esponjas; } \\
\text {-Substituição em } 42 \% \text { de material de } \\
\text { origem fóssil } \\
\text { por fonte renovável; e } \\
\text {-Aumento em quase } 200 \% \text { de } \\
\text { reciclagem de } \\
\text { material }\end{array}$ & Não informado \\
\hline \multirow[t]{3}{*}{ 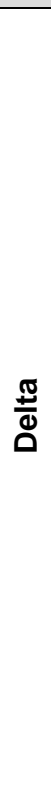 } & $\begin{array}{l}\text { Troca de tambores rotativos } \\
\text { para lavadores especiais. } \\
\text { Reuso da água de esfriamento, } \\
\text { das bombas de água de } \\
\text { alimentação da caldeira de } \\
\text { recuperação, o reuso de rejeitos } \\
\text { da osmose reversa como parte } \\
\text { do fornecimento de água } \\
\text { industrial para a fábrica, o reuso } \\
\text { de água e energia térmica } \\
\text { produzidas no digestor na } \\
\text { máquina secadora e a redução } \\
\text { no consumo de água potável. }\end{array}$ & $\begin{array}{l}\text { Diminuição de } 20 \% \text { da captação de } \\
\text { água e aumento de } 40 \% \text { da } \\
\text { reutilização de água }\end{array}$ & Não informado \\
\hline & $\begin{array}{l}\text { Para coleta, tratamento, queima } \\
\text { e controle de gases odoríferos e } \\
\text { chaminés com controle online } \\
\text { de saída dos gases }\end{array}$ & $\begin{array}{l}\text { Diminuição de mais de } 0,20 \mathrm{Kg} / \\
\text { tonelada seca à atmosfera de enxofre } \\
\text { reduzido total (ERT) de } 2005 \text { a } 2009\end{array}$ & Não informado \\
\hline & $\begin{array}{l}\text { Queima da biomassa excedente } \\
\text { da produção de } \\
\text { celulose para produção de } \\
\text { energia }\end{array}$ & $\begin{array}{l}\text { Aumento de mais de } 20 \% \text { entre } 2005 \\
\text { e } 2009 \text { de energia gerada e } \\
\text { diminuição de mais de } 400 \mathrm{KWH} / \\
\text { tonelada seca ao ar de energia } \\
\text { consumida }\end{array}$ & Não informado \\
\hline
\end{tabular}

A partir da análise do Quadro 3 é possível identificar que a $\mathrm{P}+\mathrm{L}$ traz significativos benefícios de ordem ambiental e econômica para as empresas. Sobre os indicadores ambientais da $\mathrm{P}+\mathrm{L}$, nota-se falta de mensuração exata e quantitativa na organização Alfa, contudo a empresa Beta já apresenta contabiliza seus indicadores ambientais de forma mais objetiva e exata, possibilitando assim melhor 
análise dos benefícios ambientais auferidos pela $\mathrm{P}+\mathrm{L}$. No entanto, podem-se verificar os benefícios ambientais que a $\mathrm{P}+\mathrm{L}$ promove à organização Alfa.

A organização Gama identifica os benefícios ambientais e econômicos em cada projeto de seu programa de $\mathrm{P}+\mathrm{L}$ de forma isolada dos outros sistemas de gestão e de produção. Nesta empresa existe uma campanha motivacional onde os próprios funcionários investigam possibilidades para melhorias ambientais. Esta estratégia motivacional é promovida por campanhas de competições internas em que as melhorias são premiadas.

Já a empresa Delta também mede os indicadores ambientais e econômicos dos projetos de $\mathrm{P}+\mathrm{L}$. Contudo, esta organização optou por não divulgar os resultados financeiros por questões de confidencialidade própria.

Em termos econômicos, é possível notar que a $\mathrm{P}+\mathrm{L}$ contribuiu significativamente para a redução do consumo e disposição de insumos produtivos, gerando uma notável economia financeira na aquisição de produtos e no tratamento dos resíduos produtivos. Embora o indicador econômico seja muito importante, sendo considerado o principal atrativo para a adoção da $\mathrm{P}+\mathrm{L}$, cabe ressaltar que a $\mathrm{P}+\mathrm{L}$ almeja benefícios de ordem ambiental para as organizações, sendo assim necessário que sejam levados em conta nos processos de tomada de decisão das empresas.

Os ganhos ambientais foram significativos. Foi possível observar efetivas minimizações no consumo de recursos naturais que foram possíveis devido às práticas de reutilização de recursos de produção. É importante destacar que as medidas de tratamento adotadas para posterior reutilização de recursos e água não se refere à uma prática isolada de remediação, mas sim de uma ação intermediária para que as matérias-primas possam ser reutilizadas e/ou recicladas dentro do próprio processo produtivo.

As técnicas de trocas e substituições de etapas dos processos produtivos também apresentarem importantes benefícios ambientais permitindo aumentar 0 ciclo de vida de insumos e subprodutos, minimizando parte dos impactos ambientais decorrentes destes processos de produção.

É possível identificar significativos benefícios auferidos pela $\mathrm{P}+\mathrm{L}$, contudo ainda observam-se algumas dificuldades para a sua adoção enfrentadas pelas empresas. Elas são apresentadas no Quadro 4. 
Quadro 4 - Principais dificuldades enfrentadas para a adoção da $P+L$

\begin{tabular}{|c|c|c|c|c|}
\hline & \multicolumn{5}{c|}{ Empresas } \\
\hline Dificuldadel Origem & Alfa & Beta & Gama & Delta \\
\hline Econômica & $\checkmark$ & $\checkmark$ & & $\checkmark$ \\
\hline Sistêmica & $\checkmark$ & & $\checkmark$ & \\
\hline Organizacional & & $\checkmark$ & $\checkmark$ & \\
\hline Técnica & & & & \\
\hline Comportamental & & & & \\
\hline Governamental & & & & $\checkmark$ \\
\hline
\end{tabular}

Observa-se um paradoxo enfrentado pelas organizações, pois embora o retorno financeiro seja um dos principais benefícios gerados pela $P+L$ às empresas. a falta de investimento ainda se apresenta como um obstáculo para a disseminação da $\mathrm{P}+\mathrm{L}$, pois é um aspecto determinante para a adoção ou não da $\mathrm{P}+\mathrm{L}$ pelas empresas, ou seja, antes de outros pontos importantes, as organizações priorizam a questão econômica (payback de projetos) como requisito para implantar a $\mathrm{P}+\mathrm{L}$. Esta constatação ainda pode ser realizada mesmo para projetos de $\mathrm{P}+\mathrm{L}$ que possuem complexa mensuração e perspectiva de retorno financeiro, mas com a possibilidade óbvia e clara de benefícios ambientais.

As demais dificuldades apresentadas no Quadro 4 envolvem outros aspectos de ordem sistêmica e organizacional existentes nas empresas Alfa e Beta respectivamente. A dificuldade de origem sistêmica deve-se ao fato do programa não estar inserido sistematicamente ao SGA ou departamento ambiental da empresa Alfa, pois para cada procedimento projetado, este deve ser avaliado pela área em que será implantado, principalmente no tocante ao retorno financeiro possível que este projeto possa oferecer. Desta forma, a estratégia ambiental $P+L$ é gerida fora da alçada do departamento ambiental da empresa Alfa.

A dificuldade de ordem Organizacional identificada na organização Beta é explicada pela resistência que o departamento ambiental da empresa enfrenta em conseguir apoio e autorização da diretoria para a implantação de novos projetos de $\mathrm{P}+\mathrm{L}$.

A empresa Gama não segue os passos da $P+L$ propostos pelo UNEP (2011), nem outra sistemática para a implantação de projetos e procedimentos de $\mathrm{P}+\mathrm{L}$. Neste caso observou-se dois tipos principais de dificuldades, a sistêmica e a econômica. Na organização Gama observou-se que a implantação de projetos $P+L$ é fortemente enxergado como um custo. Tal constatação pode ser explicada pela falta 
de ligação da $\mathrm{P}+\mathrm{L}$ com outras áreas da empresa realçando a falta de visão holística e sistêmica da P+L no âmbito da gestão da organização.

Como exposto no Quadro 4, a empresa Delta enfrenta dificuldades de ordem econômica e governamental. A primeira se deve ao fato de que quando não são projetos autossuficientes, existe falta de financiamentos acessíveis para a implementação das práticas ambientais. A segunda, apesar de o SGA auxiliar muito nessa parte, ainda nota-se falta de incentivo governamental, pois algumas iniciativas da empresa requerem este tipo de apoio. Esta organização consegue produzir energia elétrica por meio da transformação de energia térmica advinda da queima de biomassa em caldeiras. Esta energia é capaz de suprir toda a demanda energética da fábrica e também de grande parte da população residencial situada no município onde fica a empresa.

Embora a dificuldade de ordem governamental tenha sido observada somente na organização Delta, sabe-se que esta é um dificuldade generalizada às práticas de $\mathrm{P}+\mathrm{L}$ no âmbito empresarial nacional. Segundo UNEP (2001), as políticas públicas assumem uma vital função na disseminação das práticas de $P+L$ e seus instrumentos pode ser aplicados nas empresas em três grupos diferentes:

- regulatórios - instrumentos de caráter diretivo devem apresentar às empresas o que elas devem fazer e o que não devem fazer em relação aos seus aspectos e impactos ambientais, ressaltando o potencial da $P+L$ para este fim;

- econômicos - instrumentos de caráter incentivador devem ser sustentados por práticas de incentivos fiscais e econômicos para empresas e negócios que adotem a $\mathrm{P}+\mathrm{L}$; e

- informativos - instrumentos de caráter informativo devem, como o próprio nome diz, informar às empresas sobre as possibilidades de melhorias ambientais proativas que elas podem adotar.

\section{CONCLUSÕES}

Os estudos de caso possibilitaram concluir os objetivos propostos na pesquisa, identificando os principais benefícios auferidos pela $P+L$ às empresas $e$ 
também as principais barreiras e dificuldades para a implantação desta estratégia nas organizações do estado de São Paulo.

Destaca-se a necessidade de maior valorização dos benefícios ambientais face aos econômicos. Foi possível identificar significativos benefícios ambientais, principalmente no aspecto de redução de utilização de insumos de produção, ou seja, extração de recursos naturais e na deposição dos resíduos da produção. Além disso, a estratégia preventiva da $\mathrm{P}+\mathrm{L}$ possibilitou ganhos econômicos às organizações, sustentados pelo gerenciamento racional de seus processos produtivos. Como principal dificuldade destaca-se o aspecto econômico para a implantação de projetos de $\mathrm{P}+\mathrm{L}$.

O trabalho pode contribuir para a apresentação de significativos ganhos ambientais e econômicos que as empresas alcançam quando adotam práticas de $\mathrm{P}+\mathrm{L}$ e com isso, estimular a disseminar este programa pelo setor empresarial, pois como observado, a $\mathrm{P}+\mathrm{L}$ se apresenta como uma importante estratégia para a prevenção a poluição, minimização e mitigação de impactos ambientais nos processos produtivos, para melhoria da imagem da empresa, minimização dos custos, aumentos dos lucros, além de outros benefícios organizacionais que a $\mathrm{P}+\mathrm{L}$ promove.

Os resultados contribuem com a apresentação de referencial para futuras pesquisas utilizando outros métodos de pesquisa que permitam expandir os resultados obtidos neste trabalho. Além disso, esta pesquisa pretende estimular futuras pesquisas em sobre a $\mathrm{P}+\mathrm{L}$ que se trata de um tema importante para a área ambiental e produtiva como um todo, sobretudo ao apresentar as análises de casos de projetos de $\mathrm{P}+\mathrm{L}$ em empresas industriais diferentes.

Como limitação da pesquisa, os resultados foram identificados em quatro empresas industriais de grande porte do estado de São Paulo. Por se tratar de uma pequena amostra de pesquisa, os dados não podem ser generalizados a todo setor industrial e a outras regiões geográficas.

A realização de estudos em uma amostra maior de empresas pode contribuir para a inferência e generalização dos resultados apresentados por este artigo. A triangulação a ser proposta por meio de outros métodos de pesquisa, especialmente os quantitativos, é uma sugestão de melhorias nos vieses negativos do método de estudos de caso. 
Nota-se a necessidade de melhorias na mensuração dos benefícios ambientais, já que os econômicos são calculados detalhadamente. A necessidade de se obter um payback é a questão prioritária e até mesmo unânime nos processos de tomada de decisão das empresas que procuram implantar a $\mathrm{P}+\mathrm{L}$. A despeito da necessidade do lucro almejado pelas empresas, faz-se necessário exclamar que a $\mathrm{P}+\mathrm{L}$ é uma estratégia com cunho ambiental e este quesito não deve ser sonegado ou deixado em última instância nas tomadas de decisão das empresas. Para isso, este tema requer pesquisas que visem melhorias para sua implantação e melhor aceitação pelo meio empresarial.

\section{AGRADECIMENTOS}

Os pesquisadores agradecem a Coordenação de Aperfeiçoamento Pessoal de Nível Superior (CAPES) e ao Conselho Nacional de Desenvolvimento Científico e Tecnológico (CNPq) pelos apoios financeiros tornando possível a realização desta pesquisa.

\section{REFERÊNCIAS}

BARBIERI, J. C. Gestão ambiental empresarial: conceitos, modelos e instrumentos. 2. ed. São Paulo: Saraiva, 2007.

BAAS, L. To make zero emissions technologies and strategies become a reality, the lessons learned of cleaner production dissemination have to be known. Journal of Cleaner

Production. v. 13, p. 1205-1216, 2007. http://dx.doi.org/10.1016/j.jclepro.2006.07.017

CAGNO, E.; TRUCCO, P.; TARDINI, L. Cleaner production and profitability: an analysis of 134 pollution prevention (P2) project reports, Journal of Cleaner Production. v. 13, n. 6, p. 593-605, 2005. http://dx.doi:10.1016/i.jclepro.2003.12.025

CAUCHICK-MIGUEL, P. A. Estudo de caso na engenharia de produção: estrutura e recomendações para a sua condução. Revista Produção. v. 17, n. 1, p. 216-229, 2007. http://dx.doi.org/10.1590/S0103-65132007000100015

CENTRO NACIONAL DE TECNOLOGIAS LIMPAS (CNTL), Rio Grande do Sul. Manual de questões ambientais e Produção Mais Limpa. Apostila. Porto Alegre, 2001.

CETESB e PNUMA. A Produção mais limpa e consumo sustentável na América Latina e Caribe. Publicação das Nações Unidas. Escritório Regional para América Latina e Caribe, 2005.

CETESB. Companhia Ambiental do Estado de São Paulo http://www.cetesb.sp.gov.br/Tecnologia/producao limpa/casos geral.asp. Acessado em 05 julho 2013. 
Produção e consumo sustentáveis, 2014. Disponivel em:

http://www.cetesb.sp.gov.br/tecnologia-ambiental/Produ?o-e-Consumo-Sustent?vel/7apresentacao. Acesso em 01 jan 2014.

CHAVAN, M. An appraisal of environment management systems: a competitive advantage for small businesses. Management of Environmental Quality: An International Journal. v. 16, n. 5, p. 444-463, 2005. http://dx.doi.org/10.1108/14777830510614321

CRESWELL, J.W.: Research design: qualitative \& quantitative approaches. London : Sage, 1994.

ELIAS, S. J. B.; MAGALHÃES, L. C. Contribuição da produção enxuta para obtenção da produção mais limpa. Produção Online, v. 3, n. 4, 2003.

EPA. An Organizational Guide to Pollution Prevention, 2001. Disponível em: http://www.epa.gov/ppic/pubs/organizationalGuide.pdf. Acesso em: 13 fev. 2014.

FLEURY, A. Planejamento do projeto de pesquisa e definição do modelo teórico. In: Metodologia de pesquisa em engenharia de produção e gestão de operações. 2. ed. Rio de Janeiro: Elsevier: ABEPRO, 2012.

FRESNER, J. Cleaner production as a means for effective environmental management. Journal of Cleaner Production. v. 6, p. 171-179, 1998. http//dx. doi:10.1016/S09596526(98)00002-X

FRYXELL, G. E.; SZETO, A. The influence of motivations for seeking ISO 14001certification: an empirical study of ISO 14001 certified facilities in Hong Kong. Journal of Environmental Management. v. 65, p. 223-238, 2002.http://dx.doi:10.1006/jema.2001.0538

GRANEHEIM U. H.; LUNDMAN, B. Qualitative content analysis in nursing research: Concepts, procedures and measures to achieve trustworthiness. Nurse Education Today, v. 24, p. 105-112, 2004.

GIL, A. C. Como elaborar projetos de pesquisa. São Paulo: Atlas, 1991.

HICKS, C; DIETMAR, R. Improving cleaner production through the application of environmental management tools in China. Journal of Cleaner Production. v. 15, p. 395408, 2007. http://dx.doi:10.1016/j.jclepro.2005.11.008

HILSON, G. Barriers to implementing cleaner techonologies and cleaner production (CP) practices in the mining industry: a case study of the Americas. Minerals Engineering, v. 13, n. 7, p. 699-717, 2000. http://dx.doi:10.1016/S0892-6875(00)00055-8

HILSON, G. Defining "cleaner production" and "pollution prevention" in the mining context. Minerals Engineering, v. 16, n. 4, p. 305-321, 2003. http://dx.doi:10.1016/S08926875(03)00012-8

HUANG , Y.; JIWEN , L.; BIN , X. Application of cleaner production as an important sustainable strategyin the ceramic tile plant - a case study in Guangzhou, China. Jounal of Cleaner Production, v. 43, p. 113-121, 2013. http://dx.doi:10.1016/j.jclepro.2012.12.013 
INMETRO - INSTITUTO NACIONAL DE METROLOGIA, NORMALIZAÇÃO E QUALIDADE INDUSTRIAL 2010. Empresas certificadas ISO 14001 www.inmetro.gov.br. Acesso em: 01 mar. 2013.

IBGE. INSTITUTO BRASILEIRO DE GEOGRAFIA E ESTATÍSTICA http://www.ibge.gov.br. Acesso em: 05 out. 2013.

JUPP, V. The Sage dictionary of social research methods. London: Sage Publications, 2006.

KILBOURNE, W. E. Globalization and development: an expanded macromarketing view. Journal of Macromarketing, v. 24, n. 2, p. 122, 2004. http://dx.doi:10.1177/0276146704269303

KIST, T.; MOUTAQI, S. E.; MACHADO, L. Cleaner production in the management of water use at a poultry slaughterhouse of Vale do Taquari, Brazil: a case study. Journal of Cleaner Production, v. 17, p. 1200-1205, 2009. http://dx.doi:10.1016/j.jclepro.2009.04.006

KLEMĚS, J. J.; VARBANOV, P. S.; HUISING, D. Recent cleaner production advances in process monitoring and optimization. Journal of Cleaner Production, v. 34, p. 1-8, 2012. http://dx.doi:10.1016/j.jclepro.2012.04.026

KUBOTA, F. I.; CAMPOS, M. S.; CAUCHICK-MIGUEL, P. A. Uma análise preliminar das contribuições da modularidade em produto ao Ecodesign. Produção Online, v. 14, n. 2, p. 560-592, 2014. http://dx.doi.org/10.14488/1676-1901.v14i2.1424

LEMOS, A. D. C. A Produção mais limpa como geradora de inovação e competitividade: o caso da fazendo cerro do tigre. Dissertação (mestrado). Departamento de Administração, UFRGS. Porto Alegre, 1998.

MACIEL, D. S. C.; FREITAS, L. S. Análise do processo produtivo de uma empresa do segmento de cerâmica vermelha à luz da Produção mais Limpa. Produção Online, v. 13, n. 4, p. 1355-1380, 2013.

MITCHEL, C. L.; Beyond barriers: examining root causes behind commonly cited Cleaner Production barriers in Vietnam. Journal of Cleaner Production, v. 14, p. 1576-1585, 2006. http://dx.doi:10.1016/j.jclepro.2005.04.010

OLIVEIRA, J. A. Um estudo sobre a relação dos Sistema de Gestão Ambiental ISO 14001 com a adoção de procedimentos de Produção Mais Limpa em empresas industriais brasileiras. Dissertação (Mestrado) - Universidade Estadual Paulista. Faculdade de Engenharia, Bauru, 2011.

PNUMA. PNUMA Brasil, 2014. Disponivel em: http://www.pnuma.org.br/interna.php?id=63. Acesso em 10 jan 2014.

RIAZ, A.; ZAHEDI, G.; KLEMĚS, J. J. A review of cleaner production methods for the manufacture of methanol. Journal of Cleaner Production, v. 57, n. 15, p. 19-37, 2013. http://dx.doi:10.1016/j.jclepro.2013.06.017 
RIVELA, et al. Towards a cleaner production in developing countries: a case study in a Chilean tannery. Waste Management \& Research, v. 22, p. 131-141, Junho 2004.

RODRIGUES, M. L. M.; LIMA, R. M. F. Cleaner production of soapstone in the Ouro Preto region of Brazil: a case study. Journal of Cleaner Production, v. 32, p. 149 -156, 2012. http://dx.doi:10.1016/j.jclepro.2012.03.028

SILVA, D. A L.; DELAI, I.; CASTRO, M. A. S.; OMETTO, A. R. Quality tools applied to Cleaner Production programs: a first approach toward a new methodology. Journal of Cleaner Production, v. 47, p. 174-187, 2013. http://dx.doi:10.1016/j.jclepro.2012.10.026

SILVA, G. C. S.; MEDEIROS, D. D. Environmental management in brazilian companies. Management of Environmental Quality: An International Journal. v. 15, n. 4, p. 380-388, 2004. http://dx.doi.org/10.1108/14777830410540126 [s.n.]. 1995.

Cleaner production: a training resource package. Industry and Environment. Paris:

Cleaner production: industry and environment - seventh international high-level Seminar. Prague: [s.n.]. 2002. p. 5.

Guidance manual: how to establish and operate a cleaner production centre. Austria, 2004. Disponível em http://www.unep.fr/scp/publications/details.asp?id=WEB/0072/PA. Acesso em 12 jun 2013.

Cleaner production: industry and environment. Sixth International High-level Seminar Montreal. Canada: [s.n]. 2001.

UNIDO / UNEP. Guidance manual - how to establish and operate cleaner production centers. Cleaner Production and Environmental Management Branch. Viena. 2011.

VAN HOOF, B.; LYON, T. P. Cleaner production in small firms taking part in Mexico's Sustainable Supplier Program. Journal of Cleaner Production, v. 41, p. 270-282, 2013. http://dx.doi:10.1016/j.jclepro.2012.09.023

VOSS, C.; TSIKRIKTSIS, N.; FROHLICH, M. Case research in operations management. International Journal of Operations and Production Management. v. 22, n. 2, p. 195219, 2002. http://dx.doi.org/10.1108/01443570210414329

YOUNGBLOOD, G. J.; DVORAK, B. I.; WOLDT, W. E.; HAWKEY, S. A.; HYGNSTROM, J. R. Quantifying and comparing a P2 program's benefits: pollution prevention technical assistance in Nebraska. Journal of Cleaner Production, v. 16, p. 761-770, 2008. http://dx.doi:10.1016/j.jclepro.2007.02.016

YIN, R. K. Case study research: design and methods. 3. ed. California: Sage Publications, 2003. 
YUSUP, M. Z.; MAHMOOD, W. H. W.; SALLEH, M. R.; MUHAMAD, M. R. The Influence factor for the successful implementation of cleaner production: a review. Journal Teknologi, v. 67, n. 1, p. 89-97, 2014. http://dx.doi.org/10.11113/it.v67.2160

YÜKSEL, H. An empirical evaluation of cleaner production practices in Turkey. Journal of Cleaner Production, v. 16, n. 5, p. 0-57, 2008. http://dx.doi:10.1016/j.jclepro.2007.10.003

ZANELLI, J. C., 2002. Pesquisa qualitativa em estudos de gestão de pessoas. Estudos em Psicologia, v. 7, p. 79-88.

ZHI-DONG , L. et al. Evaluation of cleaner production audit in pharmaceutical production industry: case study of the pharmaceutical plant in Dalian, P. R. China. Clean Technology and Environmental Policy, v. 13, p. 195-206, 2011.

SENAI- Serviço Nacional de Aprendizagem Industrial. Implementação de programas de produção mais limpa. Porto Alegre, Centro Nacional de Tecnologias Limpas SENAI-RS/ UNIDO/INEP, 42 p., 2003.

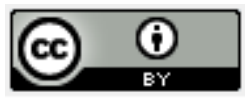

Artigo recebido em 29/05/2014 e aceito para publicação em 13/04/2015 DOI: http://dx.doi.org/ 10.14488/1676-1901.v15i2.1751 